\title{
Desempeño Reproductivo de Vacas Lecheras con Involución Uterina Retardada bajo Tratamiento Hormonal con Cipionato de Estradiol y Benzoato de Estradiol
}

\author{
Reproductive Performance of Dairy Cows with Delayed Uterine Involution \\ under Hormonal Therapy with Estradiol Cypionate and Estradiol Benzoate
}

\author{
Luis Felipe Ruiz García ${ }^{1}$, Rocío Silvia Sandoval Monzónn, \\ Milena Montenegro Vega ${ }^{1}$, Alfredo Delgado Castro ${ }^{1}$
}

\section{RESUMEN}

\begin{abstract}
El objetivo de este estudio fue evaluar el desempeño reproductivo de vacas lecheras con involución uterina retardada y sometidas a dos tratamientos hormonales (cipionato de estradiol $\left[\mathrm{CE}_{2}\right]$ y benzoato de estradiol $\left[\mathrm{BE}_{2}\right]$ ) entre los 36 y 49 días después del parto y un grupo de vacas con cuernos uterinos involucionados sin tratamiento. Se trabajó con 349 vacas con involución uterina retardada y 127 vacas con cuernos involucionados de un establo lechero comercial en Lima, Perú. El BE $\mathrm{B}_{2}$ se aplicó en 3 dosis de 5 mg por animal, vía i.m. interdiaria y el $\mathrm{CE}_{2}$ se aplicó en 2 dosis de $4 \mathrm{mg}$ por animal, vía i.m. en un intervalo de 4 días. Se evaluó el intervalo parto concepción (IPC), el número de servicios por concepción (NSC), la tasa de concepción al primer servicio (P1S) y el porcentaje de preñez a los 200 días (P200d). Además, se evaluó la probabilidad de que las vacas queden preñadas en función de los días en lactación, empleando el modelo de riesgos proporcionales de Cox. El IPC y el NSC fueron menores en vacas tratadas con $\mathrm{CE}_{2}$ (122.1 días y 1.3, respectivamente) que en las tratadas con $\mathrm{BE}(138.4$ días y 1.5 , respectivamente $)(\mathrm{p}<0.05)$. Asimismo, la P1S y el P200d fueron similares en las vacas con $\mathrm{CE}_{2}$ (49.3 y 47.7\%, respectivamente) y las tratadas con $\mathrm{BE}_{2}$ (36.7 y $50.2 \%$, respectivamente). Las vacas tratadas con CE tuvieron 1.6 veces más probabilidades de preñar más pronto que las vacas tratadas con $\mathrm{BE}_{2}(\mathrm{p}<0.01)$. Se recomienda la aplicación de cipionato de estradiol sobre el benzoato de estradiol para mejorar el desempeño reproductivo de vacas lecheras con involución uterina retardada.
\end{abstract}

Palabras clave: involución uterina retardada, vacas lecheras, tratamiento, desempeño reproductivo

\footnotetext{
${ }^{1}$ Clínica de Animales Mayores, Facultad de Medicina Veterinaria, Universidad Nacional Mayor de San Marcos, Lima, Perú

${ }^{2}$ E-mail: rociossandovalm@gmail.com
}

Recibido: 2 de julio de 2016

Aceptado para publicación: 12 de noviembre de 2016 
The aim of this study was to evaluate the reproductive performance of dairy cows with delayed uterine involution under hormonal treatment with estradiol cypionate $\left(\mathrm{CE}_{2}\right)$ and estradiol benzoate $\left(\mathrm{BE}_{2}\right)$ between 36 and 49 days after parturition and a group of dairy cows with normal uterine involution. A total of 349 dairy cows with delayed uterine involution and 127 dairy cows without delayed uterine involution from a commercial farm in Lima, Peru were used. The BE treatment consisted of the i.m. application of 3 doses of $5 \mathrm{mg}$ at 2-day interval and the $\mathrm{CE}_{2}$ treatment consisted of the i.m. application of 2 doses of $4 \mathrm{mg}$ at 4-day interval. The parameters evaluated were calving-to-conception interval (IPC), services per conception (NSC), conception rate at first service (P1S) and the pregnancy rate at 200 days (P200D). In addition, the likelihood of get pregnant according to the days in lactation using Cox proportional hazards model. Both the IPC and the NSC were lower in cows treated with $\mathrm{CE}_{2}$ (122.1 days and 1.3 respectively) that in cows treated with $\mathrm{BE}_{2}$ (138.4 days and 1.5 , respectively $(\mathrm{p}<0.05)$. The P1S and P200D were similar in cows with $\mathrm{CE}_{2}$ (49.3 and $47.7 \%$ respectively) and in cows treated with $\mathrm{BE}_{2}$ (36.7 and 50.2\% respectively). In addition, cows treated with $\mathrm{CE}_{2}$ were 1.6 times more likely to conceive sooner than cows treated with $\mathrm{BE}_{2}(\mathrm{p}<0.01)$. The application of estradiol cypionate in relation to oestradiol benzoate is recommended to improve the reproductive performance in dairy cows with delayed uterine involution.

Key words: delayed uterine involution, dairy cows, treatment, reproductive performance

\section{INTRODUCCIÓN}

Las infecciones uterinas son trastornos comunes que afectan a las vacas lecheras durante el periodo posparto. Existe amplia información en la literatura científica indicando que las infecciones uterinas producen un retardo de la involución uterina, lo cual se traduce en el desmedro del desempeño reproductivo (Wagner et al., 2001; Risco y Hernández, 2003). Pese a su importancia, todavía existen divergencias de opiniones entre veterinarios respecto al diagnóstico y tratamiento de las infecciones uterinas (Risco et al., 2007).

Si bien los aspectos relacionados a la reproducción del hato dependen de múltiples factores (nutrición, genética, medio ambiente, manejo, etc.) (Lewis, 1997), los tratamientos preventivos y curativos aplicados durante el puerperio tienen un efecto trascendente sobre el desempeño reproductivo (Sheldon y Noakes, 1998; Overton et al., 2003). El éxito de estos tratamientos permite reducir el periodo voluntario de espera e incrementar la fertilidad al primer servicio, lo que significa que se puede lograr una concepción más temprana (Wagner et al., 2001; Risco y Hernández, 2003).

Las bacterias ambientales contaminan el lumen uterino durante el parto, en más del $90 \%$ de las vacas; sin embargo, la respuesta inmune es capaz de eliminar esta contaminación bacteriana durante las primeras cinco semanas después del parto (Sheldon et al., 2003). Cuando la contaminación persiste, se produce la enfermedad o infección uterina que trae como consecuencia la involución uterina retardada, prolongando el intervalo parto-primer servicio y parto-concepción (LeBlanc et al., 2002a). La involución uterina retardada es uno de los factores que limita la fertilidad en la vaca (Senger, 2003, Sheldon et al., 2004a), aunque algunos autores han encontrado ligera asociación entre la involución uterina retardada y el desempeño reproductivo (LeBlanc et al., 2002b). 
Las enfermedades uterinas más comunes son metritis, endometritis y piometra (Sheldon et al., 2006a). La endometritis es la forma menos severa y más común de las infecciones uterinas. Se caracteriza por la inflamación del útero sin presentación de signos clínicos sistémicos (Lewis, 1997; LeBlanc et al., 2002a; Sheldon et al., 2006a). En general, las enfermedades uterinas no solo causan infertilidad al momento de la infección, sino que además pueden resultar en subfertilidad, aun cuando haya resolución clínica de la enfermedad (Azawi, 2008).

El tratamiento de la infección uterina en la especie bovina es un tema bastante polémico. Se han descrito tratamientos intrauterinos con antibióticos y químicos antisépticos, tratamientos con antibióticos sistémicos, así como terapias hormonales (Hussain y Daniel, 1991). Una variedad de hormonas ha sido administrada a vacas, en un intento de prevenir o tratar las infecciones uterinas después del parto. Por ejemplo, la oxitocina se ha utilizado con la finalidad de mejorar la evacuación del contenido uterino, aunque esta hormona solo produce contracción del miometrio cuando el órgano está dominado por los estrógenos; de allí que puede ser efectiva si se administra dentro de las 48 a 72 horas después del parto (Risco et al., 2007).

Por otra parte, los estrógenos se han aplicado con la finalidad de iniciar y fortalecer las contracciones del miometrio, pero su uso es controversial (Risco et al., 2007; Azawi, 2008). El estradiol estimula la reepitelización y vascularización del endometrio (Noakes et al., 2001), incrementa la producción de moco cervical y secreciones del oviducto, mejora la contractibilidad del útero (Ali et al., 2003) y tiene efectos específicos en el sistema inmune secretor (Wira y Rossoll, 1995); sin embargo, la información disponible no es concluyente (Wagner et al., 2001; Overton et al., 2003; Risco y Hernández, 2003).
En los establos lecheros de Lima, se ha difundido el uso de esteres de estradiol (benzoato y cipionato de estradiol), con la finalidad de prevenir y tratar las infecciones uterinas que producen un retardo de la involución uterina. No obstante, no existen estudios que demuestren la eficacia de ninguno de estos tratamientos en el país. Por lo tanto, el objetivo del presente estudio fue evaluar el efecto de los esteres de estradiol en el tratamiento de las infecciones uterinas en vacas lecheras.

\section{Materiales y Métodos}

\section{Lugar de Estudio}

El estudio fue realizado en un establo lechero de crianza intensiva de la provincia de Lima, departamento de Lima, Perú. El establo contaba con 1200 vacas Holstein, de la cuales cerca de 900 se encontraban en ordeño durante el periodo de estudio. Las vacas se encontraban alojadas en corrales en grupos de 50 animales aproximadamente, según la paridad (primíparas o multíparas) y días en lactación (DEL).

Las vacas paridas en un año (365 días) fueron revisadas ginecológicamente entre los 36 y 49 días del parto. Se incluyeron en el estudio todas las vacas que fueron diagnosticadas con involución uterina retardada y aquellas con cuernos uterinos involucionados al momento de la revisión ginecológica.

El periodo voluntario de espera (PVE) del establo era de 60 días, a partir del cual eran inseminadas las vacas que mostraban celo. La inseminación artificial (IA) era realizada con semen congelado en pajillas de 0.5 $\mathrm{ml}$. Todas las IA fueron realizadas siguiendo las condiciones estándares de trabajo. La detección de celos era visual y las hembras era inseminadas siguiendo el protocolo AM/ PM. El diagnóstico de preñez fue realizado mediante palpación rectal entre los 35 y 45 
días después de la inseminación sin retorno de celo.

\section{Tamaño Muestral}

Se empleó la fórmula para el cálculo del tamaño de muestra en estudios de supervivencia (Pértegas y Pita, 2002):

$$
n=\frac{\left(z_{1-\alpha / 2}+z_{1-\beta}\right)^{2}}{(\log (R R))^{2}(1-\psi)(1-p) p}
$$

donde $\mathrm{n}$ es el tamaño muestral que se quiere calcular; Z1-á/2 es el nivel de confianza o seguridad con el que se desea trabajar, en este caso 1.96 (á = 95\%); Z1-â es el poder que se quiere para el estudio, en este caso $0.842(\hat{a}=80 \%)$; $\log (\mathrm{RR})$ es el logaritmo natural del valor aproximado del riesgo relativo (RR) que se desea detectar, en este caso 1.4; $p$ es la proporción de expuestos al factor de estudio, en este caso es la proporción de animales tratados con benzoato de estradiol (50\%); Ø es el porcentaje de observaciones censuradas que se espera en el total de la muestra, en este caso $15 \%$. El tamaño muestral mínimo resultante fue de 113 vacas por grupo.

\section{Diseño del Estudio}

Este fue un estudio de cohortes, por lo cual se realizó el seguimiento de los animales hasta los 200 DEL a las vacas diagnosticadas con involución uterina retardada y con cuernos involucionados entre los 36 y 49 días posparto. Todos los diagnósticos fueron hechos por el mismo médico veterinario. El criterio de diagnóstico de involución uterina retardada se basó en el hallazgo por palpación rectal de asimetría notoria entre los cuernos, grosor de uno o ambos cuernos superior a los $35 \mathrm{~mm}$ de diámetro y sin contenido en el útero ni presencia de descargas purulentas o mucopurulentas por la vagina (Pimentel, 2001; LeBlanc et al., 2002a; Ruizy Sandoval, 2013). Se registró el número de parto, la fecha del último parto, los hallazgos a la palpación rectal, el número de servicios, la fecha del servicio efectivo y los tratamientos correspondientes.
Se formaron tres grupos de estudio de acuerdo al tratamiento que recibieron las vacas:

- Sin tratamiento (ST): Las vacas que presentaron cuernos uterinos involucionados en la revisión ginecológica. Asimismo, no se les aplicó tratamiento antibiótico u hormonal antes de superado el periodo voluntario de espera.

- Benzoato de estradiol (BE): Las vacas que presentaron involución uterina retardada en la revisión ginecológica. Se les inyectó benzoato de estradiol (i.m.) en dosis de $5 \mathrm{mg}$ por animal por tres veces con intervalos de 2 días.

- Cipionato de estradiol (CE): Las vacas que presentaron involución uterina retardada en la revisión ginecológica. Se les inyectó cipionato de estradiol (i.m.) en dosis de $4 \mathrm{mg}$ por animal, aplicado en dos oportunidades con intervalos de 4 días.

Se excluyeron del estudio todas las vacas que presentaron algún problema de enfermedad metabólica o infecciosa grave, las vacas con endometritis o piometra al momento de la revisión ginecológica y las que recibieron tratamiento diferente a los anteriormente indicados. Asimismo, las observaciones fueron retiradas cuando las vacas murieron, fueron descartadas o se descontinuó el estudio.

\section{Variables de Interés}

Porcentaje de preñez acumulada a los 200 días (P200d). Representa la oportunidad de que las vacas queden preñadas durante los primeros 200 días posparto. Fue calculado por la división del número de vacas preñadas antes de los 200 días del parto entre el número total de vacas, expresado en porcentaje (Ruiz y Sandoval, 2014).

Tasa de concepción al primer servicio (P1S). Se define como la oportunidad de que una vaca inseminada quede preñada al primer servicio. Fue calculada por la división 
del número de vacas preñadas al primer servicio entre el número de vacas que recibieron uno o más servicios, expresado en porcentaje (Fetrow et al., 1990).

Intervalo parto - concepción (IPC). Representa el tiempo transcurrido desde el parto hasta la concepción (Fetrow et al., 1990).

Número de servicios por concepción (NSC). Representa el número de servicios necesarios para poder conseguir una preñez (Fetrow et al., 1990).

\section{Análisis Estadístico}

El análisis estadístico de los parámetros reproductivos se realizó empleando el modelo lineal generalizado. E1 IPC se analizó utilizando la distribución normal y como función de enlace identidad, para el NSC se utilizó la distribución de Tweedie y como función de enlace logaritmo, mientras que para la P1S y el P200d se empleó la distribución binomial y como función de enlace logit. Como variables independientes en el modelo, se analizaron la época de parto (verano, otoño, invierno, primavera), condición corporal al momento de la revisión ginecológica $(<2.75 v s \geq 2.75)$, paridad (primíparas $v s$ multíparas) y tratamiento ( $\left.\mathrm{ST}, \mathrm{CE}_{2}, \mathrm{BE}_{2}\right)$.

La probabilidad de que las vacas queden preñadas en función de los días posparto fue evaluada usando el modelo de regresión de riesgos proporcionales de Cox (Pinedo, 2011). Las variables independientes utilizadas para el análisis fueron el tratamiento, la estación de parto, la condición corporal al momento de la revisión ginecológica y la paridad. Los valores beta estimados, el error estándar del beta estimado y los riesgos relativos (RR) ajustados fueron reportados.

Para el análisis de regresión de riesgos relativos de Cox, se utilizaron como variables de referencia el grupo $\mathrm{BE}_{2}$ para el tratamiento, la primavera para la estación de parto, las vacas con una puntuación menor de 2.75 para la condición corporal y las vacas multíparas para la paridad. El riesgo relativo fue usado como una medida epidemiológica de asociación entre las variables independientes (tratamiento o covariables) y la variable dependiente (probabilidad de quedar preñada en función de los DEL).

Para el análisis estadístico se empleó el software IBM SPSS Statistics 22. En todos los análisis se consideró como significativa una probabilidad menor de $5 \%$.

\section{Resultados}

La distribución de las vacas, según paridad, época de parto, condición corporal y tratamiento, se muestra en el Cuadro 1.

El efecto de los tratamientos sobre los parámetros reproductivos se puede apreciar en el Cuadro 2. El IPC y el NSC fueron significativamente mejores en las vacas tratadas con cipionato de estradiol, en comparación con las vacas tratadas con benzoato de estradiol $(\mathrm{p}<0.05)$. Del mismo modo, las vacas tratadas con cipionato de estradiol necesitaron un menor número de servicios para quedar preñadas en comparación con las vacas con cuernos involucionados que no recibieron tratamiento $(p<0.05)$, aunque no se encontró diferencia estadística significativa entre estos grupos para el IPC. Por otro lado, no hubo diferencia estadística entre los tres grupos para P1S y P200d.

En el Cuadro 3 se presentan los resultados del análisis de riesgo proporcional de Cox para los días a la concepción. La paridad no tuvo efecto significativo en el modelo, mientras que la época de parto, la condición corporal y los tratamientos para la involución uterina retardada presentaron diferencias estadísticas $(p<0.05)$. Las vacas tratadas con $\mathrm{CE}_{2}$ tuvieron una mayor probabilidad de preñar $(R R=1.63)$ que las vacas tratadas con $\mathrm{BE}_{2}(\mathrm{p}<0.001)$, del mismo modo que las vacas con cuernos involucionados sin tratamiento $(R R=1.33, p<0.05)$. En forma similar, las 
Cuadro 1. Distribución de las vacas según las variables en estudio

\begin{tabular}{|c|c|c|}
\hline Características & Total & Porcentaje \\
\hline \multicolumn{3}{|l|}{ Época } \\
\hline Verano & 112 & 23.5 \\
\hline Otoño & 99 & 20.8 \\
\hline Invierno & 132 & 27.7 \\
\hline Primavera & 133 & 27.9 \\
\hline \multicolumn{3}{|l|}{ Condición corporal } \\
\hline$<2.75$ & 146 & 30.7 \\
\hline$>2.75$ & 330 & 69.3 \\
\hline \multicolumn{3}{|l|}{ Paridad } \\
\hline Primípara & 192 & 40.3 \\
\hline Multípara & 284 & 59.7 \\
\hline \multicolumn{3}{|l|}{ Grupo } \\
\hline Sin tratamiento & 127 & 26.7 \\
\hline Cipionato de estradiol & 149 & 31.3 \\
\hline Benzoato de estradiol & 200 & 42.0 \\
\hline Total & 476 & 100.0 \\
\hline
\end{tabular}

Cuadro 2. Parámetros reproductivos ${ }^{1}$ (promedio \pm intervalo de confianza) de vacas Holstein $(\mathrm{n}=476)$ con involución uterina normal o retardada al diagnóstico ginecológico a los 36-49 días del parto, se gún el tratamiento recibido

\begin{tabular}{lcccccccc}
\hline Grupo & \multicolumn{2}{c}{ IPC } & \multicolumn{2}{c}{ NSC } & \multicolumn{2}{c}{ P1S } & \multicolumn{2}{c}{ P200d } \\
\cline { 2 - 8 } & $\mathrm{n}$ & Prom \pm I.C. & $\mathrm{n}$ & Prom \pm I.C. & $\mathrm{n}$ & $\% \pm$ I.C. & $\mathrm{n}$ & $\% \pm$ I.C. \\
\hline $\begin{array}{l}\text { Sin } \\
\text { tratamiento }\end{array}$ & 80 & $129.6 \pm 6.5^{\mathrm{b}}$ & 80 & $1.56 \pm 0.12^{\mathrm{a}}$ & 126 & $37.5 \pm 8.5^{\mathrm{a}}$ & 127 & $56.7 \pm 8.6^{\mathrm{a}}$ \\
$\begin{array}{l}\text { Cipionato de } \\
\text { estradiol }\end{array}$ & 95 & $122.1 \pm 7.0^{\mathrm{b}}$ & 95 & $1.29 \pm 0.11^{\mathrm{b}}$ & 131 & $49.3 \pm 8.6^{\mathrm{a}}$ & 149 & $47.7 \pm 8.0^{\mathrm{a}}$ \\
$\begin{array}{l}\text { Benzoato de } \\
\text { estradiol }\end{array}$ & 88 & $138.4 \pm 5.5^{\mathrm{a}}$ & 88 & $1.47 \pm 0.12^{\mathrm{a}}$ & 173 & $36.7 \pm 7.2^{\mathrm{a}}$ & 200 & $50.2 \pm 6.9^{\mathrm{a}}$ \\
\hline 1
\end{tabular}

1 IPC: Intervalo parto concepción; NSC: Numero de servicios por concepción; P1S: Tasa de concepción al primer servicio; P200d: Porcentaje de preñez a los 200 días del parto

${ }^{a, b}$ Superíndices diferentes dentro de columnas indican diferencia significativa $(p<0.05)$ 
Cuadro 3. Resultados del análisis de riesgo proporcional de Cox para los días a la concepción

\begin{tabular}{|c|c|c|c|c|c|c|c|c|}
\hline & \multirow{2}{*}{$\beta^{1}$} & \multirow{2}{*}{$\mathrm{ET}^{2}$} & \multirow{2}{*}{ Wald $^{3}$} & \multirow{2}{*}{$\mathrm{gl}^{4}$} & \multirow{2}{*}{ Sig. ${ }^{5}$} & \multirow{2}{*}{$\mathrm{RR}^{6}$} & \multicolumn{2}{|c|}{ 95\% IC para RR } \\
\hline & & & & & & & Inferior & Superior \\
\hline Época de parto & & & 28.80 & 3 & 0.00 & & & \\
\hline Primavera & Ref. & & & & & & & \\
\hline Verano & 0.45 & 0.17 & 7.01 & 1 & 0.01 & 1.58 & 1.13 & 2.21 \\
\hline Otoño & 0.99 & 0.19 & 28.07 & 1 & 0.00 & 2.69 & 1.87 & 3.88 \\
\hline Invierno & 0.43 & 0.16 & 7.75 & 1 & 0.01 & 1.55 & 1.14 & 2.10 \\
\hline \multicolumn{9}{|c|}{ Condición corporal } \\
\hline$<2.75$ & Ref. & & & & & & & \\
\hline$\geq 2.75$ & 0.27 & 0.12 & 4.64 & 1 & 0.03 & 1.30 & 1.02 & 1.66 \\
\hline Tratamiento & & & 10.18 & 2 & 0.01 & & & \\
\hline Control & 0.29 & 0.13 & 4.54 & 1 & 0.03 & 1.33 & 1.02 & 1.73 \\
\hline Cipionato & 0.49 & 0.16 & 9.69 & 1 & 0.00 & 1.63 & 1.20 & 2.21 \\
\hline Benzoato & Ref. & & & & & & & \\
\hline \multicolumn{9}{|l|}{ Paridad } \\
\hline Primípara & Ref. & & & & & & & \\
\hline Multípara & -0.13 & 0.12 & 1.13 & 1 & 0.29 & 0.88 & 0.70 & 1.11 \\
\hline
\end{tabular}

vacas paridas en primavera tuvieron una menor probabilidad de preñar $(R R=1)$ en comparación con aquellas paridas en verano $(\mathrm{RR}=1.58 ; \mathrm{p}<0.05)$, invierno $(\mathrm{R} R=1.55$; $\mathrm{p}<0.05)$ y otoño $(\mathrm{RR}=2.69 ; \mathrm{p}<0.01)$. Además, las vacas con condición corporal mayor o igual a 2.75 presentaron una mayor probabilidad de preñar $(\mathrm{RR}=1.30)$ en comparación con las vacas con condición corporal menor de 2.75 $(\mathrm{p}<0.05)$.

\section{Discusión}

En el caso del bovino, la involución uterina debe completarse entre 3 y 5 sema- nas después del parto, donde pasado este tiempo debería encontrarse un útero normal a la palpación rectal (Sheldon et al., 2006a; Ruiz y Sandoval, 2013). La palpación de cuernos engrosados entre los 39 y 49 días posparto indica un retardo en la involución uterina (Sheldon y Noakes, 1998; Ruiz y Sandoval, 2013). Aunque la palpación rectal no permite un diagnóstico definitivo, sigue siendo un método práctico y económico para detectar el retardo de la involución uterina en las vacas lecheras (Hazen et al., 2000; Pimentel, 2001; Sheldon et al., 2006b).

Los resultados obtenidos muestran que el tratamiento con $\mathrm{CE}_{2}$ mejoró significa- 
tivamente algunos de los parámetros reproductivos en comparación con $\mathrm{BE}_{2}$. Esto puede deberse a la mayor vida media del cipionato en relación con el benzoato (Driowo et al., 1980; Kuhl, 1990; Vynckier et al., 1990), lo que prolongaría el efecto estrogénico sobre el útero. Además, las vacas con involución uterina retardada tratadas con $\mathrm{CE}_{2}$ tuvieron un desempeño reproductivo similar al de las vacas con cuernos involucionados en las que no se empleó tratamiento alguno.

El CE es formado por la esterificación del estradiol por el ácido propiónico ciclopentano, lo que resulta en una menor solubilidad en el agua y, consecuentemente, una liberación más lenta desde el sitio de administración y una actividad biológica más prolongada en comparación al $\mathrm{BE}_{2}$ (Kuhl, 1990; Vynckier et al., 1990). Asimismo, el pico de la hormona luteinizante (LH) ocurre a las 19.6 horas de la aplicación de $\mathrm{BE}_{2}$, en tanto que ocurre a las 50.5 horas de la aplicación del $\mathrm{CE}_{2}$ (Sales et al., 2012).

En el análisis de supervivencia, las vacas tratadas con $\mathrm{CE}_{2}$ tuvieron un $50 \%$ más de probabilidades de preñar en menor tiempo que las vacas tratadas con $\mathrm{BE}_{2}$. No se han encontrado estudios que comparen el efecto a largo plazo de estos tratamientos; sin embargo, el cipionato ha sido usado con poco éxito para prevenir infecciones uterinas pocos días después del parto (Wagner et al., 2001; Overton et al., 2003; Risco y Hernández, 2003). Asimismo, LeBlanc et al. (2002b) no encontraron un efecto benéfico sobre la tasa de concepción posparto al tratar las endometritis antes de las cuatro semanas posparto. En forma similar, Sheldon y Noakes (1998), aplicando CE a los 21 días posparto, no encontraron diferencias en la tasa de curación entre los animales tratados con $\mathrm{BE}_{2}$, oxitetraciclina intrauterina y $\mathrm{PGF}_{2 a}$.

Las vacas paridas en primavera tuvieron una menor probabilidad de preñar que las paridas en otras épocas del año. Esto está en concordancia con lo encontrado por Evaristo y Echevarría (1999) en Lima, y se explica porque estas vacas, luego del periodo voluntario de espera de 60 a 90 días se inseminan en verano con un índice de estrés de calor mayor de $72\left(27-32{ }^{\circ} \mathrm{C}\right.$ y $80 \%$ de humedad relativa), que es considerado como estrés térmico para vacas lecheras (Armstrong, 1994). El estrés térmico afecta la tasa de concepción al producir en las vacas un patrón irregular en la secreción de estradiol o la elevación de la temperatura de ambiente uterino, que causarían mayor mortalidad embrionaria temprana (De Rensis y Scaramuzzi, 2003).

\section{Conclusión}

El tratamiento de la involución uterina retardada a los 36-49 días del parto en vacas Holstein con cipionato de estradiol mejoró los parámetros reproductivos estudiados en relación con el tratamiento con benzoato de estradiol.

\section{Literatura Citada}

1. Ali A, Abdel-Razek A, Abdel-Ghaffar S, Glatzel P. 2003. Ovarian follicular dynamic in buffalo cows (Bubalus bubalis). Reprod Dom Anim 38: 214218. doi: 10.1046/j.1439-0531.2003.00428.x

2. Armstrong D. 1994. Heat stress interaction with shade and cooling. J Dairy Sci 77: 2044-2050. doi: 10.3168/ jds.S0022-0302(94)77149-6

3. Azawi OI. 2008. Postpartum uterine infection in cattle. Anim Reprod Sci 105: 187-208. doi: 10.1016/j.anireprosci.2008.01.010

4. De Rensis F, Scaramuzzi R. 2003. Heat stress and seasonal effects on reproduction in the dairy cow - a review. Theriogenology 60: 1139-1151. doi: 10.1016/S0093-691X(03)00126-2

5. Driowo MA, Landgren BM, Stenström B, Diczfalusy E. 1980. A comparison of the pharmacokinetic properties of three estradiol esters. Contraception 21: 
415-424. doi: 10.1016/S0010-7824(80)80018-7

6. Evaristo R, Echevarría L. 1999. Factores que afectan el intervalo parto primer servicio en vacas lecheras de crianza intensiva. Rev Inv Vet Perú 10: 22-26. doi: 10.15381/rivep.v10i2.6699

7. Fetrow J, McClary D, Harman R, Butcher K, Weaver L, Studer E, Ehrlich J, et al. 1990. Calculated selected reproductive indices: recommendations of the American Association of Bovine Practitioners. J Dairy Sci 73: 78-90. doi: 10.3168/ jds.S0022-0302(90)78649-3

8. Hanzen CH, Pieterse M, Scenczi O, Drost M. 2000. Relative accuracy of the identification of ovarian structures in the cow by ultrasonography and palpation per rectum. Vet J 159: 161-170. doi: 10.1053/tvj1.1999.0398

9. Hussain AM, Daniel RCW. 1991. Bovine endometritis: current and future alternative therapy. Zentralbl Veterinarmed A 38: 641-651. doi: 10.1111/ j.1439-0442.1991.tb01060.x

10. Kuhl H. 1990. Pharmacokinetics of oestrogens and progestogens. Maturitas 12: 171-197. doi: 10.1016/03785122(90)90003-O

11. LeBlanc SJ, Duffield TF, Leslie KE, Bateman KG, Keefe GP, Walton JS, Johnson WH. 2002a. Defining and diagnosing postpartum clinical endometritis and its impact on reproductive performance in dairy cows. J Dairy Sci 85 : 2223-2236. doi: 10.3168/jds.S00220302(02)74302-6

12. LeBlanc SJ, Duffield TF, Leslie KE, Bateman KG, Keefe GP, Walton JS, Johnson WH. 2002b. The effect of treatment of clinical endometritis on reproductive performance in dairy cows. J Dairy Sci 85: 2237-2249. doi: 10.3168/ jds.S0022-0302(02)74303-8

13. Lewis GS. 1997. Uterine health and disorders. J Dairy Sci 80: 984-994. doi: 10.3168/jds.S0022-0302(97)76024-7
14. Noakes D, Parkinson T, England G, Arthur G. 2001. Arthur's veterinary reproduction and obstetrics. $8^{\text {a }}$ ed. USA: Saunders-Elsevier. $868 \mathrm{p}$.

15. Overton M, Sischo W, Reynolds J. 2003. Evaluation of effect of estradiol cypionate administered prophylactically to postparturient dairy cows at high risk for metritis. J Am Vet Med Assoc 223: 846-851. doi: $10.2460 /$ javma.2003.223.846

16. Pértegas S, Pita S. 2002. Cálculo del tamaño muestral para la determinación de factores pronósticos. [Internet]. Disponible en: http://www.fisterra.com/mbe/ investiga/muestra_pronos/ pronosti.asp\#supervivencia

17. Pimentel CA. 2001. Infertilidade na fêmea bovina. En: Riet-Correa F, Schild AL, Mendez MC, Lemos RAA (eds). Doenças dos ruminantes e eqüinos. Vol 2. $2^{\text {a }}$ ed. São Paulo, Brasil: Ed Varela. p 361-379.

18. Pinedo PJ. 2011. Applied statistical analyses for dairy production. En: Risco CA, Retamal PM(eds). Dairy production medicine. UK: Blackwell Publishing. p 263-270.

19. Risco CA, Hernández J. 2003. Comparison of ceftiofur hydrochloride and estradiol cypionate for metritis prevention and reproductive performance in dairy cows affected with retained fetal membranes. Theriogenology 60:4758. doi: 10.1016/S0093-691X(02)01299-2

20. Risco C, Youngquist R, Shore M. 2007. Postpartum uterine infections. En: Youngquist R, Threlfall W (eds). Current therapy in large animal - Theriogenology. $2^{\text {nd }}$ ed. Missouri: Saunders Elsevier. $p$ 339-344.

21. Ruiz LF, Sandoval RS. 2013. Involución uterina en el ganado bovino: Un nuevo score para su evaluación y su relación con el número de partos y los días en lactación. Spermova 3: 87-88.

22. Ruiz LF, Sandoval RS. 2014. Relación entre los parámetros reproductivos convencionales y los parámetros de eficien- 
cia reproductiva de los establos lecheros de Lima. Spermova 4: 58-60.

23. Sales J, Carvalho J, Crepaldi G, Cipriano R, Jacomini J, Maio J, Souza $J$, et al. 2012. Effects of two estradiol esters (benzoate and cypionate) on the induction of synchronized ovulations in Bos indicus cows submitted to a timed artificial insemination protocol. Theriogenology 78: 510-516. doi: 10.1016/ j.theriogenology.2012.02.031

24. Senger P. 2003. Pathways to pregnancy and parturition. $2^{\text {nd }}$ ed. USA: Current Conceptions. 373 p.

25. Sheldon IM, Barrett DC, Boyd $H$. 2004a. The postpartum period. En: Andrews AH, Blowey RW, Boyd H, Eddy RG (eds). Bovine medicine diseases and husbandry of cattle. $2^{\text {nd }} e d$. UK: Blackwell Science. p 508-529.

26. Sheldon IM, Lewis GS, LeBlanc S, Gilbert RO. 2006a. Defining postpartum uterine disease in cattle. Theriogenology 65: 1516-1530. doi: 10.1016/j.theriogenology.2005.08.021

27. Sheldon IM, Wathes DC, Dobson H. $2006 \mathrm{~b}$. The management of bovine reproduction in elite herds. Vet J 171: 7078. doi: 10.1016/j.tvj1.2004.06.008
28. Sheldon IM, Noakes DE, Rycroft AN, Dobson H. 2003. The effect of intrauterine administration of estradiol on postpartum uterine involution in cattle. Theriogenology 59: 1357-1371. doi: 10.1016/S0093-691X(02)01169-X

29. Sheldon IM, Noakes DE. 1998. Comparison of three treatments for bovine endometritis. Vet Rec 142: 575 579. doi: 10.1136/vr.142.21.575

30. Vynckier L, Debackere M, De Kruif A, Coryn M. 1990. Plasma estradiol$17 \beta$ concentrations in the cow during induced estrus and after injection of estradiol-17 $\beta$ benzoate and estradiol-17 $\beta$ cyprionate - a preliminary study. J Vet Pharmacol Ther 13: 36-42.

31. Wagner DC, BonDurant RH, Sischo WM. 2001. Reproductive effects of estradiol cypionate in postparturient dairy cows. J Am Vet Med Assoc 219: 220 223. doi: 10.2460/javma.2001.219.220

32. Wira CR, Rossoll RM. 1995. Antigen presenting cells in the female reproductive tract: Influence of estrus cycle on antigen presentation by uterine epithelial and stromal cells. Endocrinology 136: 4526-4534. doi: 10.1210/endo.136.10.7664673 